\title{
3 Zielsetzung und Motivation
}

Wie bereits in Kapitel 2.4 beschrieben, kann der fluiddynamische Mehrkoordinatenantrieb in zwei Bewegungsrichtungen ( $x$ und $y$ ) frei positioniert werden. Da der Antrieb über keinerlei mechanische Führungselemente verfügt, wird die Drehung des Tisches um die z-Achse regelungstechnisch durch die Einleitung von Momenten über die Antriebselemente gesperrt. Es konnte gezeigt werden, dass der vorhandene Prototyp bei Mittelstellung des Tisches bereits geregelte Drehbewegungen um die zAchse ermöglicht. Diese sind jedoch auf maximal $\pm 15^{\circ}$ begrenzt, da es bei größeren Winkeln zu ungünstigen Überdeckungsverhältnissen von Profilflanken und Antriebsdüsen kommt. Das zentrale Ziel dieser Arbeit ist nun die Entwicklung einer Profil- und Düsenanordnung, die diese Begrenzung aufhebt und damit den bestehenden Antrieb um einen vollwertigen Rotationsfreiheitsgrad erweitert. Ein solcher Planarantrieb, dessen Läufer in jeder Position des Verfahrbereichs unbeschränkte Drehfreiheit erlaubt, ermöglicht eine weitere Steigerung der funktionellen Integrationsdichte. Auf diese Weise wird ein wesentlicher Ansatz, den das SPP 1476 zur Reduzierung der Baugröße von Werkzeugmaschinen vorsieht, konsequent weiter verfolgt.

Weitere Ziele bestehen in der Optimierung der neuen Profilanordnung hinsichtlich

- maximaler Antriebskräfte und Momente,

- minimalem Bauraum und

- minimaler Düsenzahl.

Dabei soll der grundlegende Aufbau des Antriebs und das Wirkprinzip zur Krafterzeugung jedoch beibehalten werden. Das erste Optimierungskriterium ergibt sich direkt aus den Eigenschaften des bestehenden Demonstrators und dessen relativ geringen Vorschubkräften. Die beiden folgenden Kriterien resultieren aus dem erklärten Ziel des SPP 1476 und aus Überlegungen zur Kosten- bzw. Komplexitätsreduzierung.

Als abschließendes Ergebnis dieser Arbeit wird die Ausarbeitung einer konstruktiv umsetzbaren Profilanordnung angestrebt, die später in einem neuen Demonstrator eingesetzt werden kann. 